$\xi=-1$

\title{
Overview of Device-to-Device Communication and Vehicle-to-Vehicle Communication
}

\author{
H. Srikanth Kamath ${ }^{1 *}$, Sreelakshmi ${ }^{2}$, Muthyala Siri Chandana Reddy ${ }^{3}$, Chelsea Camilo Monteiro ${ }^{4}$ \\ ${ }^{1}$ Assistant Professor, Manipal Institute of Technology, Manipal-576104, Karnataka, India \\ ${ }^{2}$ Manipal Institute of Technology, Manipal - 576104, Karnataka, India \\ ${ }^{3}$ Manipal Institute of Technology, Manipal - 576104, Karnataka, India \\ ${ }^{4}$ Manipal Institute of Technology, Manipal-576104, Karnataka, India \\ *Corresponding author E-mail: srikanth.kamath@manipal.edu
}

\begin{abstract}
5G (5th generation mobile networks or 5th generation wireless systems) is the next major phase of mobile telecommunications standards beyond the current 4G LTE (Long-Term Evolution) standards. 5G technology needs to be specified, developed, and deployed by a variety of industry players including network equipment vendors, network operators, semiconductor vendors, and device manufacturers. The scope of $5 \mathrm{G}$ will range from mobile phones to next-generation automobiles. Device to Device (D2D) Communication is regarded as a promising technology in 5G to provide low power, high data rate and low latency. Introducing D2D poses many challenges and risks to the longstanding cellular architecture, which is centred on the base station.
\end{abstract}

Keywords:

\section{Introduction}

Device-to-device (D2D) communication technology for fifth generation $(5 \mathrm{G})$ mobile networks is one in which user equipment (UE) can communicate directly with other UEs with limited association of the network infrastructure like mobile access points or base stations. At present we have low level D2D techniques such as Bluetooth and WiFi Direct. These can only be used for short range transmission since they operate at low frequency licenseexempt bands. For the implementation of Device to Device communication foreseen for $5 \mathrm{G}$ networks, there is a need for a fundamental change in the design structure.

Looking at its architecture, the D2D communication system is very alike to Mobile Ad-hoc networks (MANETs) and Cognitive Radio Network (CRN). A MANET is a group of wireless mobile hosts which form a temporary network without any help of a centralized administration or standard support services [2]. Its applications are military communications, emergency situations and meetings. Cognitive radio is an intelligent, adaptive radio and network technology that automatically detects wireless channels in a wireless spectrum and instantly moves to them while avoiding occupied ones. Both of these are generally multi hop networks.

\section{Current Scenario}

In the present $4 \mathrm{G}$ or LTE (Long Term Evolution) scenario, we can see that there is one cellular base station, or evolved Node-B (eNB) which controls all the cellular devices. With Device-toDevice Communication, we aim to connect more devices to the system hence extending the network to areas where it is difficult to set up base stations. This will allow us to allocate more resources without much tradeoff in network speeds.

$4 \mathrm{G}$ systems use a frequency band of $2-8 \mathrm{GHz}$. $5 \mathrm{G}$ is proposed to use a frequency band of $15 \mathrm{GHz}$. This means that transmission will be faster but it will have a shorter range.

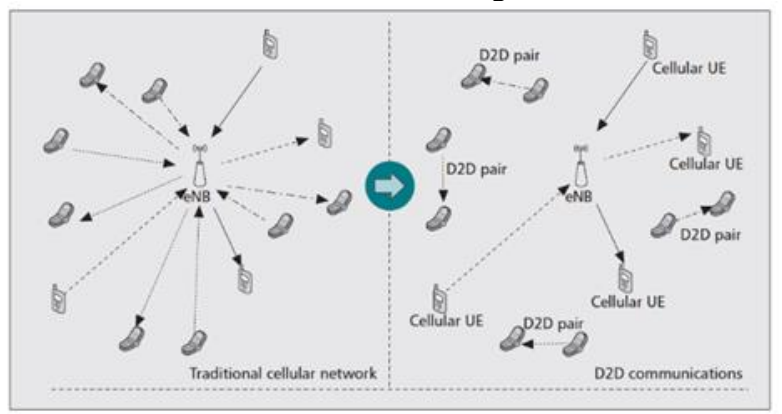

Fig. 1. Traditional Cellular vs. D2D

\section{D2D Modes and Resource Blocks}

Different modes of D2D provide good throughput. Information is usually transferred in form of resource blocks. The different modes are:

- Silent Mode: When the resources that are available are not enough for spectrum reuse, the devices cannot communicate and remain silent.

- Reuse Mode: D2D devices transmit data be reusing the spectrum of other cellular users. The reuse of spectrum can either be in downlink or uplink mode. 
- Dedicated Mode: The cellular network dedicates a portion of its spectrum for D2D transmission only. Hence the devices can transmit information without much interference from other users.

- Cellular Mode: The D2D devices communicate in the same way as traditional cellular communication through an eNB.

Most of these information is sent as a cluster of resource blocks. A resource block is the smallest unit of resources. One block typically is made up of resource elements. How much information is transmitted by these elements depends on the modulation scheme used. For example, a resource element with QPSK modulation has a capacity of 2 bit. If it uses 16QAM scheme then it can occupy 4 bits.

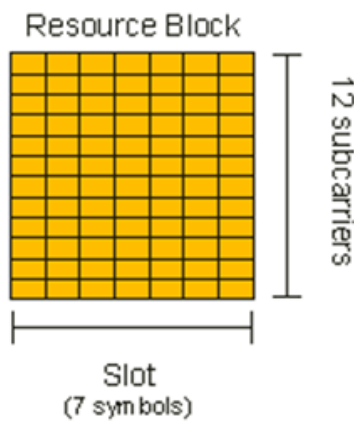

Fig. 2. Resource Block

\section{Outline for device to device communication}

In the architecture perspective, D2D communication networks observed to be similar to Mobile Ad-hoc Networks (MANETs) and also Cognitive Radio Network (CRN). Mobile Ad-hoc network is a collection of wireless mobile hosts forming a temporary network without the aid of any centralized administration or standard support services. They are mostly used in emergency situations, military communications and meetings. Cognitive radio is an adaptive, intelligent radio and network technology that can automatically detect wireless channels in a wireless spectrum and instantly move to them while avoiding occupied ones. These both are generally multi hop networks. D2D communication can be divided into two parts:

- In-band D2D: In this category, the communication takes place in the licensed spectrum. There is a belief that interference in the unlicensed band is hard to manage.

- Out-band D2D: Unlike in-band, this category works in unlicensed spectrum. This category requires an extra interface and generally uses the existing technologies like Zigbee, Wi-Fi and Bluetooth.

Device to device communication can be either base station controlled or device controlled. Therefore, in the device tier, device to device communication is classified into four types:

- Device relaying with operator controlled link establishment (DR-OC): If there is a device in a poor coverage area or on the cell edge, it is capable of communicating with the BS by relaying its information through other devices. Operator communicates with the relay devices for the link establishment Higher QoS and more battery power can be achieved through this. The architecture is shown in Fig 3.

- Direct device to device communication with operator controlled link establishment (DC-OC): The devices exchange the data with each other directly even without the help of base station or the relays. The devices take the help of operator for the establishment of control links. The architecture is shown in the Fig 4.

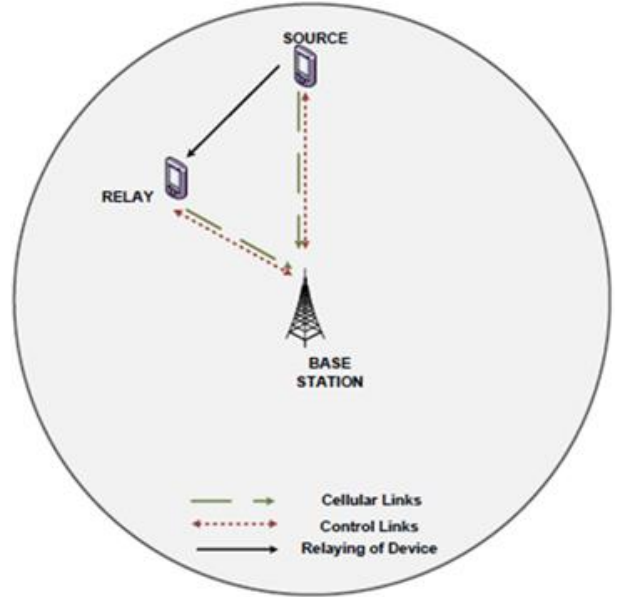

Fig. 3. Device relaying with operator controlled link

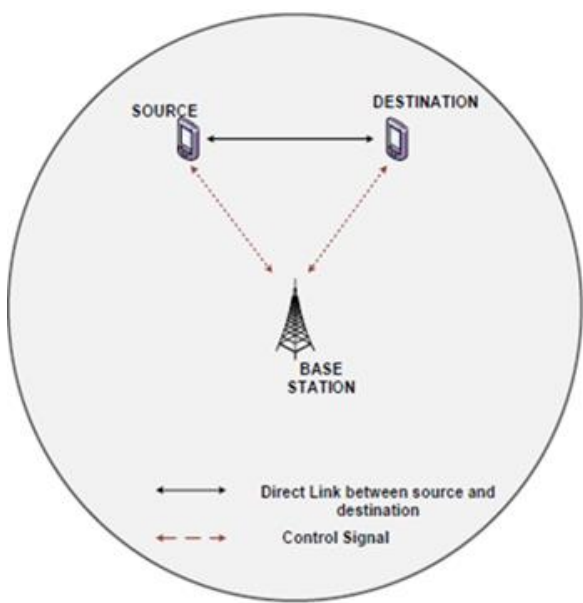

Fig. 4. Direct communication between devices with operator controlled link establishment

- Device relaying with device controlled link establishment (DR-DC): The operator is not involved in the process of communication. The devices coordinate the communication between each other using the relays. The architecture is shown in the Fig 4

- Device to device communication with device controlled link establishment (DC-DC): Devices communicate with each other without the help of operator. Resource allocation and interference management are controlled by the devices itself. The architecture is shown in the Fig. 5.

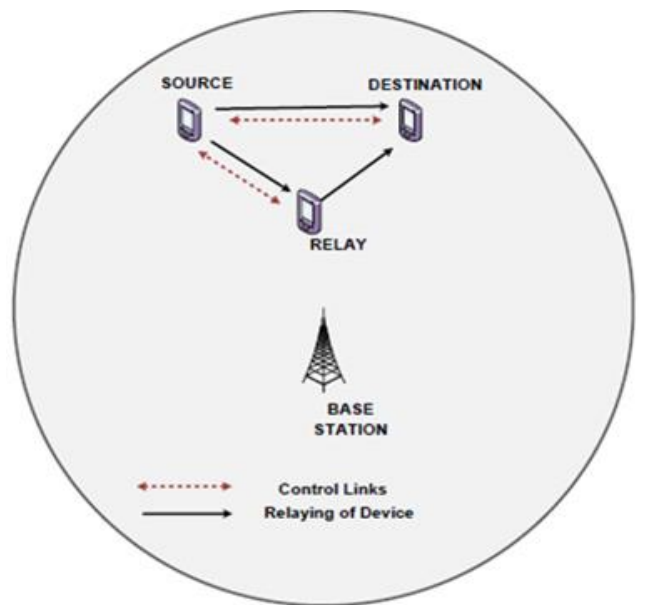

Fig. 5. Device relaying with device controlled link establishment 


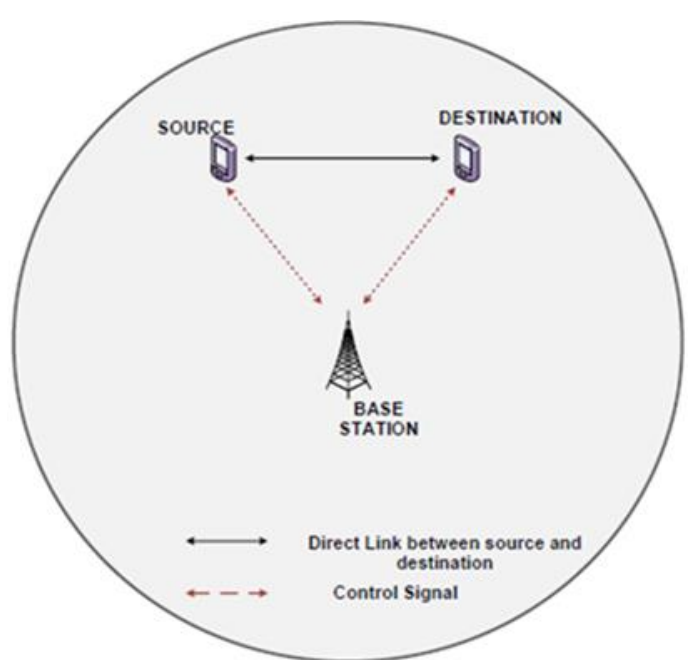

Fig. 6. Direct device to device communication device controlled link establishment.

The device to device communication has more advantages than the existing cellular networks. Some of the benefits are:

- Single hop communication: The devices will be able to communicate with each other through a single hop. Since the devices communicate directly with each other, latency is reduced. Lesser resources are used resulting in efficient utilization of the spectrum.

- Optimization of Power Levels: Since the device to device communication depends on the proximity and takes place in lower distance, the transmission power is less. This enhances the battery life of the devices and also efficient usage of energy

- Enhanced Coverage Area: As discussed, using the relays can support communication over greater ranges and thereby improving the overall coverage area.

\section{Features of D2D}

Few of the key features of device to device communication which help in achieving the required target are listed below:

\subsection{Millimeter wave}

Providing connectivity in the dense node deployment scenarios has signified the need for spectrum management. Millimeter wave spectrum band $(30-300 \mathrm{GHz})$ is being considered as a viable option for future 5G D2D [3]. This technology provides gains in terms of low interference and high data rates. Standardization is required due to the frequency rand of unlicensed millimetre band. We can only reap its benefits if the networks could be leveraged, design issues are identified and standard specifications are determined. The range of millimeter wave in the electro-magnetic spectrum is considered to be from 10 millimeters to 1 millimeter. It can be observed that millimeter waves are longer than infrared waves and $\mathrm{x}$-rays, but shorter than the radio waves and microwaves. The frequency range of millimeter wave in electromagnetic spectrum corresponds to $30 \mathrm{GHz}$ to $300 \mathrm{GHz}$. Millimeter wave range is also referred to as Extremely high frequency (EHF) range. More bandwidth is available in the millimetre wave and hence higher data rates are achieved.

\subsection{Cooperative Communication}

Cooperative communication is one of the fastest growing areas of research, and it is likely to be a key enabling technology for efficient spectrum use in future. When the devices are far away from each other, cooperative communication comes into picture. Since the relays are used, the selection of relays has to be optimal and efficient. For the selection of relays, the base stations play a key role. Though the cooperative communication improves the system performance and QoS, the user equipment power is extremely consumed and this needs to be optimised.

\subsection{Massive MIMO}

Massive MIMO is the physical layer technology for future wireless access. The main concept is to use larger antenna arrays at both transmitter and receiver side. It provides better throughput and spectrum efficiency.

\subsection{Hybrid Automatic Repeat Request (HARQ) Opera- tion}

HARQ is a combination of automatic repeat request and forward error correction. This is supposed to make D2D more robust. HARQ with a combination of ARQ and 'soft combining' is generally used. In the soft combining technique, the received error data is not discarded anymore, instead it is stored in buffer and will be combined in next retransmission. 


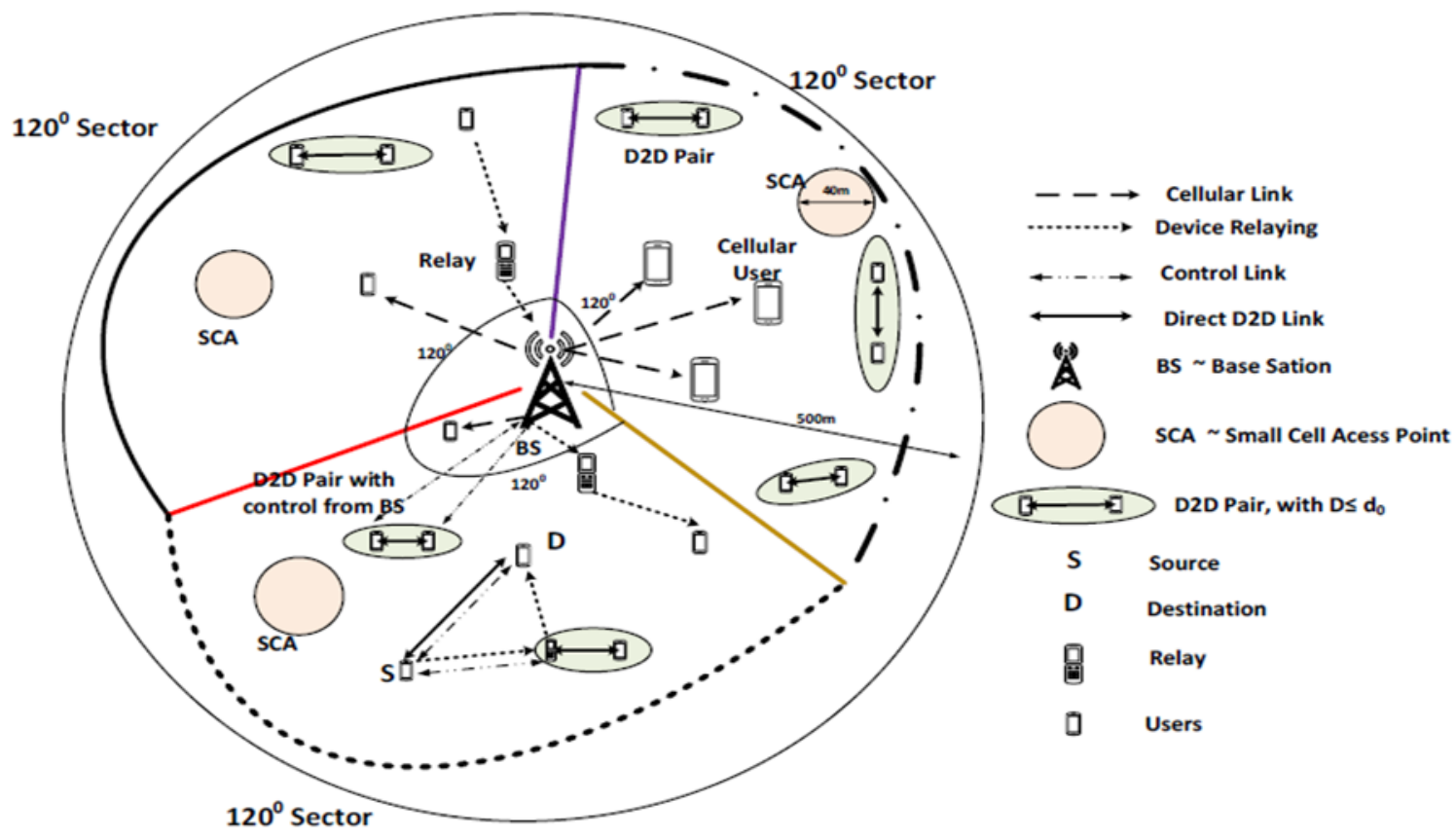

Fig. 7. Proposed Architecture

\subsection{Cognitive Radio}

Cognitive networks play a major role in effective utilization of spectrum. They have a class of networks which have the ability to change their transmission parameters, thereby enabling more communications to run concurrently. It is a software defined network. In SDN, single radio is supposed to work on different bands, frequencies or technologies. There are limitations for CRNs. There is no complete automation and it requires user intervention for changes to be implemented. There is great security concern. The data may be eavesdropped or altered without any notice data is decoded. For this to happen the destination node needs to be synchronised with the transmitter node. Though it has advantages, large amount of resources are to be used. Due to merging, there is a chance of losing uniqueness of each packet.

\subsection{Network Coding}

Network coding is a networking technique in which the transmitted data is encoded and decoded to increase network throughput and make the network more robust. Unlike the conventional way, the packets which are supposed to go for the same destination are merged using some algorithms and then sent through the routing node. At the destination, the received data is decoded. For this to happen the destination node needs to be synchronised with the transmitter node. Though it has advantages, large amount of resources are to be used. Due to merging, there is a chance of losing uniqueness of each packet.

\section{Key Challenges}

In this section we will discuss the key challenges that are associated with device to device communication

- Resource Allocation

There are three modes which are common, based on which the base station decides to allocate resources to the devices [5].

- Cellular Mode: The D2D users communicate which each other normally using base station like in current scenarios. The resources are divided equally among the cellular users as well as the D2D users.
- Dedicated Resource Mode: The D2D users communicate with each other through dedicated resource allocated by the base station. This is also known as orthogonal sharing because no resource is reused. The remaining resources are divided amongst the other cellular users.

- Reuse Resource of one cellular user: The D2D pair re uses the transmission link of one of the cellular devices which has the smallest interference between it. This is known as nonorthogonal sharing.

- Reuse Resource of more than one cellular user: There is a minimum guaranteed data rate and the D2D pair can reuse the resources of more than one cellular user.

The first three methods are what is already proposed. The 4th method is a new way for resource allocation which shows an improved throughput result compared to reusing the resource only of one user.

The base station calculates the maximum throughput allowed in the above four cases using the Shannon capacity formula and hence selects the mode with maximum throughput for optimal resource allocation.

The increasing number of subscribers and need for high data rates, there is a need for greater number of channels per unit coverage area of the cell. As a result we use cell sectoring. We split the cells into sectors by using directional antennas instead of an omnidirectional antenna at the base station. This enhances the channel capacity. Each sector operates at its own frequency level, reducing the interference and improving the Signal to Interference Noise ratio.

In model Fig 6, the cell is sectored into 3 sectors. Each sector can have any number of users. This facilitates efficient device-todevice communication (D2D) by reducing the load on the main base station hence providing it with better resource allocation as each sector is allowed to use more resource than compared with a single omnidirectional antenna. The UEs close to the base station will operate normally but those away will use D2D techniques depending on the distance between them. The architecture aims at maximizing throughput, minimizing latency, enhancing system capacity, and efficiently utilizing the licensed spectrum through optimal resource allocation.

- Peer Discovery

This process should be efficient as the popularity for D2D communication is gaining massive popularity. Another reason for the increased efficiency is because to discover the D2D links and 
establish them. The process of setting up these links needs to discover the respective devices first. The method of discovery could be elaborated on the basis of different perspectives.

From the user's perspective, they are classified into:

- Restricted mode: UEs cannot be detected unless they give the permission beforehand. This mode is thus very crucial in maintaining privacy and is mainly used in social network applications like group gaming, context sharing among a group etc.

- Open discovery mode: In this type of mode, UEs can be detected during the duration in which they lie in the proximity of the other UEs. This mode is suitable for public safety purposes, for example when the network coverage is unavailable as in the situation like when a natural disaster had occurred.

From the power consumption perspective, a low power consumption scheme is proposed [7]. They can be classified into:

- Location information-based discovery: In this, the users report their current location information, which will be obtained by the location-measuring apparatus, to the location management server. The neighbours can be recognized by the server using collected information. This type is usually used in social media services, where the respective setting of an application will be changed according to the region they are in.

- Proximity beacon-based discovery scheme: In this, the peers can be detected by a predefined proximity beacon. Each users broadcast their own proximity beacon at a specific time to notify the others of their existence in that duration of proximity.

A major disadvantage of Location information-based discovery scheme is that the user has to constantly update their location. This increases power consumption and computational overhead. Location management server usually breaks down due to huge loads involved in managing all the information of the network. Proximity beacon-based discovery scheme has a higher advantage in terms of power consumption which can be greatly reduced by varying the accuracy and scope of peer detection. There is usually a tradeoff between them. For example, users can switch to sleep mode without receiving beacons from other users in order to save energy, but might miss the presence of these users. Furthermore, users in close could use a low power consumption if the transmission of the beacon can be scheduled in more precise way. Users can hence reduce power consumption by adjusting the accuracy and scope of peer detection.

- Interference Management One of the main challenges is the interference from the cellular users. This management is mainly concentrated on the under laid networks as it consists of both D2D and cellular users and lead to more interference issues. The user suffers from inter-cell and intra-cell interference. It depends on the operation mode of the D2D network, i.e. uplink and downlink. Several interference management techniques have been proposed in various studies, some of them are interference avoidance, interference coordination, and interference cancellation.

- Interference avoidance: Here, the transmission is manipulated to avoid interaction between interfering nodes. An interference limited area (ILA) based approach for interference avoidance is used. In this, a geographical area around the D2D user is defined. The cellular users falling within that area are not allowed to transmit simultaneously with the D2D users. The extent of ILA depends on the comparison of interference-tosignal ratio values at the $\mathrm{D} 2 \mathrm{D}$ receiver with a predetermined threshold. Also two D2D pairs are not allowed to simultaneously transmit.

- Interference coordination: This scheme gain significant relevance in in band D2D communication. It can be classified into 2 parts:

- Centralized interference coordination (CIC): It involves the supervision of base station.
○ Decentralized interference coordination (DIC): It involves the participation of D2D nodes with minimal supervision of base station.

- Interference cancellation: In this technique the receiver is allowed to decode the message by mitigating the impact of interference.

- Power Control

Cellular links suffer from high interference from other user equipment and the best way to control it would be to control the power in the uplink channels. We can do this by setting a maximum power level for every D2D transmitter. The level can be chosen so that the expected degradation level remains at a tolerable level. But the drawback is that it will have to be designed for a worst case scenario which will result in wastage of resources.

\section{Security}

The exchange of information between D2D users is more vulnerable due to the exposed nature of wireless communication. A secure communication must satisfy requirements of authenticity, privacy, confidentiality, integrity and availability [6]. The following security requirements for D2D communication is highlighted.

- Authentication: It is important to protect D2D communication from impersonate attacks. The D2D system should be able to verify, whether a D2D user is allowed to use the D2D services or not. The authenticity between legitimate D2D users enables us to uniquely identify each other. This helps to distinguish between authorized users and unauthorized users.

- Non repudiation: It is important to know the source of a message that a receiver receives. An attacker could act innocent and impose the innocent party as the attacker. If non repudiation is guaranteed, the receiver would be able to verify the source whether it is legit or not.

- Secure routing and transmission: In presence of adversaries, a user should be able to transmit information in a secured manner. It should be ensured that only intended users would be able to read any exchanged content.

- Confidentiality: A D2D service should control the data access to ensure that only authenticated user can access it.

- Integrity: The goal here is to ensure the transfer of legit and real content instead of falsification and modification of any content that is exchanged.

\section{Applications}

The most important application for D2D communication is cellular offloading and hence increased network capacity. Device-todevice communication can be used for multicasting and in public safety announcement systems. In case of an emergency, D2D communication can play an essential role. If the cellular networks (base stations) are damaged during a natural calamity, then a wireless network can be setup using D2D communication to help communicate. Social apps will now be able to use proximity features identifying other users nearby. D2D combined with IoT can support many applications such as vehicle-to-vehicle communication. This is important in collision avoidance systems. There are a wide variety of applications offered for the next generation networks by device-to-device communication.

\section{Vehicle-to-Vehicle Communication}

Vehicle-to-vehicle communication ( $\mathrm{V} 2 \mathrm{~V}$ communication) is the wireless transmission of data between motor vehicles. The main 
goal of this type of communication is to prevent accidents by allowing vehicles to send their current location and speed data to one another over an ad hoc mesh network [16]. Depending upon how exactly the technology is implemented, the vehicle's driver may simply receive a warning if there is a risk of an accident or the vehicle itself may take proactive actions such as braking to slow down. It is expected to be better than the current automotive original embedded manufacturers in lane departure, adaptive cruise control, blind spot detection, rear parking sonar and backup camera because V2V technology enables a 360-degree awareness of surrounding threats. The implementation of a V2V communication system in a vehicle would be costly but it would be efficient and it could bring about a whole new insight into the advancement of technology [9].

\subsection{Capacity for V2V Communication}

We calculated the ergodic capacity of vehicle to vehicle communication and how it varies with speed. We plotted the sum capacity against varying speeds. We used 2 Algorithms for resource allocation, where algorithm one maximises the sum capacity and algorithm 2 maximizes the minimum capacity [12].

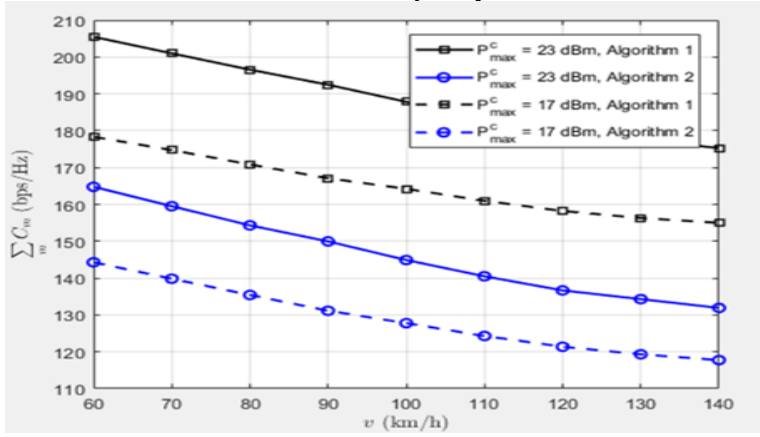

Fig. 8. Sum capacity against varying speeds

Here we can see that the sum capacity, which is depicted by algorithm 1 gives higher result than the minimum capacity algorithm. This is so because algorithm 1 aims to maximize the sum capacity. We can also observe that an increase in the transmit power has a relatively constant impact on sum CUE performance in both algorithms 1 and 2 with respect to vehicle speed increase.

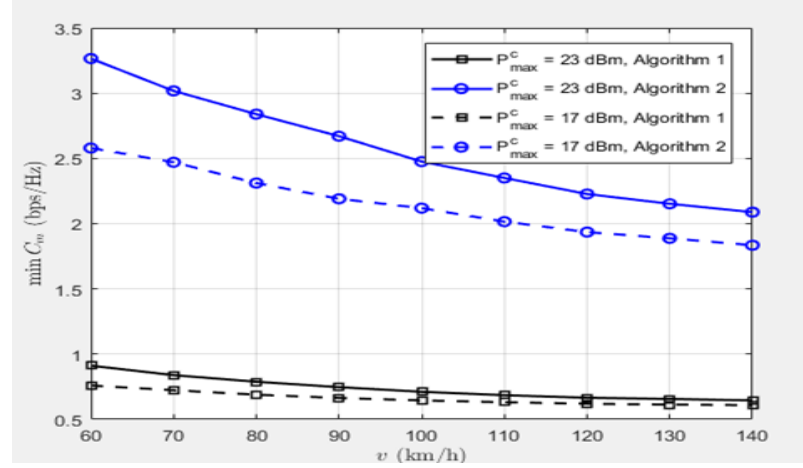

Fig. 9. Minumum capacity against varying speeds

In fig 9, we can see that the minimum capacity which is depicted by algorithm 2 produces a higher result. This is because algorithm 2 aims to maximize the sum capacity. At low vehicle speeds, a $6 \mathrm{dBm}$ increase in transmit power shows gains for both algorithms, but at high speeds it's almost constant.

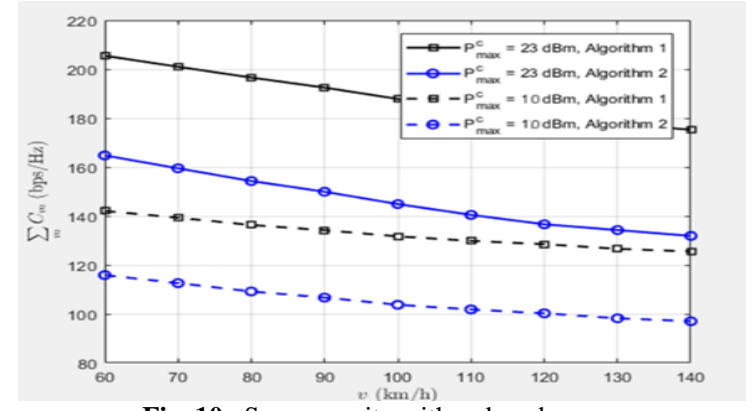

Fig. 10. Sum capacity with reduced power

We also plotted the Sum CUE ergodic capacity with a lower maximum transmit power, i.e. $10 \mathrm{dBm}$ and observed that in this case, we cannot choose any one algorithm and say which is better since the $10 \mathrm{dBm}$ of algorithm 1, produces lower capacity than the $23 \mathrm{dBm}$ of algorithm 2 .

\section{Conclusion}

We can conclude from the results we have obtained that device to device communication shows a very promising future. It can provide speeds of up to $1 \mathrm{Gbps}$ while tackling the current problem of lack of bandwidth availability.

However, in vehicle to vehicle communication, a lot more needs to be researched on how it can be made more practical for a traffic scenario. From our simulations, we observed that the capacity for vehicles can be adjusted by adjusting the transmit power. No one algorithm gives a greater result.

\section{References}

[1] Mohsen Nader, Tehrani, Murat Uysal and Halim Yanikomeroglu, "Device to Device Communication in 5G Cellular Networks: Challenges, Solutions and Future Directions ", IEEE Community Magazine, vol: 52, issue: 5, pp 86-92, May 2014

[2] Pimmy Gandotra, Student Member, IEEE, Rakesh Kumar Jha Member, IEEE, "Device-to-Device Communications in Cellular Networks: A Survey" in the Journal of Network and Computer Applications, vol 71, pp 99-117, August 2016

[3] Rafay Iqbal Ansari, Chrysostomos Chrysostomou, Syed Ali Hassan, Mohsen Guizani, Shahid Mumtaz, Jonathan Rodriguez, Joel J. P. C. Rodrigues, "5G D2D Networks: Techniques, Challenges, and Future Prospects" IEEE Systems Journal, vol:1, issue: 99, pp 1-15, December 2017.

[4] T. Laxminidhi, V. Prasadu, and S. Pavan, "Widely Programmable High-Frequency Active RC Filters in CMOS Technology", IEEE Trans. Circuits and Systems-I: Regular Papers, vol.56, pp. 327-336, 2009.

[5] Bing Wang, Li Chen, Xiaoheng Chen, Xin Zhang, Dacheng Yang, "Resource Allocation Optimization for Device-to-Device Communication Underlaying Cellular Networks", Vehicular Technology Conference (VTC Spring), 2011 IEEE 73 ${ }^{\text {rd }}$, Yokohama, Japan, May 2011.

[6] Michael Haus, Muhammad Waqas, Aaron Yi Ding, Yong Li,, Sasu Tarkoma,, Jörg Ott, "Security and Privacy in Device-toDevice(D2D) Communication: A Review", IEEE Communications Surveys and Tutorials, January 2017

[7] Woongsup Lee, Juyeop Kim, Sang-Won Choi, "New D2D Peer Discovery Scheme based on Spatial Correlation of Wireless Channel", IEEE Transactions on Vehicular Technology, December 2016

[8] Daquan Feng, Lu Lu, Yi Yuan-Wu, Geoffrey Ye Li, Shaoqian Li, and Gang Feng, "Device-to-Device Communications in Cellular Networks", IEEE Communications Magazine, vol: 52, issue: 4, pp 49-55, April 2014

[9] J. Kapusta and A. Kalašová, "Motor Vehicle Safety Technologies in Relation to the Accident Rates," Communications in Computer and Information Science Tools of Transport Telematics, pp. 172179, 2015.

[10] A.S. Khan, Yasir Javed, J. Abdullah, J.M. Nazim, N. Khan, "Security issues in 5G device to device communication", IJCSNS International Journal of Computer Science and Network Security, vol.17, no.5, May 2017 
[11] Phond Phunchongharn, Ekram Hossain and Dong In Kim, "Resource allocation for device-to-device communications underlaying LTE-advanced networks", IEEE Wireless Communications vol: 20, issue: 4, pp 91-100, August 2013

[12] Le Liang, Student Member, IEEE, Geoffrey Ye Li, Fellow IEEE and Wei Xu, Senior Member, IEEE, "Resource Allocation for D2D-Enabled Vehicular Communications", IEEE Transactions on Communications, vol: 65, issue: 7, pp 3186 - 3197, July 2017. 